\title{
Variables de Influencia en la Capacidad de Aprendizaje. Un Análisis por Conglomerados y Componentes Principales
}

\author{
Laura Gallego ${ }^{1}$ y Oscar Araque $^{2}$ \\ (1) Facultad de Contaduría Pública, Universidad Cooperativa de Colombia, sede Ibagué - Espinal 730001, \\ Colombia (e-mail: laura.gallego@campusucc.edu.co) \\ (2) Departamento de Ingeniería Mecánica, Universidad de Ibagué, Ibagué 730001, Colombia \\ (e-mail: oscar.araque@unibague.edu.co)
}

Recibido Jul. 4, 2018; Aceptado Sep. 24, 2018; Versión final Nov. 20, 2018, Publicado Abr. 2019

\begin{abstract}
Resumen
Este artículo tiene como objetivo determinar la correlación existente entre las variables que potencian la capacidad de aprendizaje asociada al desarrollo de la investigación en las Instituciones de educación superior. Con tal propósito se utiliza la técnica multivariada de análisis de conglomerados de variables y se construye el clúster jerárquico utilizando el software SPSS. A partir del método ward para agrupación de clúster e intervalo de distancia euclídea cuadrado, se identifican los grupos que se forman. Esta selección de conglomerados se corrobora con la técnica multivariada de análisis de componentes principales, con un análisis de matriz de correlaciones de KMO (Kaiser, Meyer y Olkin) y prueba de esfericidad de Bartlett. El análisis mostró que la capacidad de aprendizaje presenta una fuerte interacción con el sector externo, reflejado por el alto nivel de relación entre alianzas y políticas institucionales y de estos con el desarrollo de convenios. La capacitación y formación de posgrado de los investigadores y la vigilancia de los saberes presentan un menor nivel de correlación con las demás variables.
\end{abstract}

Palabras clave: instituciones de educación; investigación; capacidades de aprendizaje; SPSS; estadística

\section{Variables of influence in the learning capability. An analysis by Clusters and main components}

\begin{abstract}
The purpose of this manuscript is to determine the correlation between the variables that enhance the learning capability associated with the development of research in Higher Education Institutions. For this purpose, the multivariate analysis of clusters of variables using the SPSS software is applied. From the ward method for cluster grouping and square Euclidean distance interval, the groups that are formed are identified. This selection of clusters is corroborated with the multivariate technique of principal component analysis, with an analysis of KMO correlation matrix (Kaiser, Meyer and Olkin) and Bartlett's sphericity test. The analysis showed that the learning capability presents a strong interaction with the external sector, reflected by the high level of relationship between alliances and institutional policies and of these with the development of agreements. The training and graduate training of researchers and the monitoring of knowledge present a lower level of correlation with the other variables.
\end{abstract}

Keywords: educational institutions; research; learning capability; SPSS; statistics 


\section{INTRODUCCIÓN}

La capacidad de una organización para afrontar los problemas que se le presentan y la posibilidad de aprovechar a su favor el entorno es una forma adecuada de definir las Capacidades Dinámicas (CD), estas a su vez pueden llegar a ser comunes en muchos tipos de organizaciones y se desarrollan permitiendo una evolución de tal forma que permean la estructura de la misma, pero a la vez deben ser lo suficientemente flexibles para permitir su adaptabilidad (Helfaf y Peteraf, 2015). Las capacidades dinámicas deben estar acompañadas de un manejo apropiado de los recursos, estos recursos son el conjunto de insumos que tiene una organización y a través de éstos se desempeñan las actividades y tareas de la misma, de acuerdo al investigador Grant (1991), es una herramienta que permite determinar las fortalezas y debilidades internas de la organización. Una de las definiciones mayormente aceptada ha sido propuesta por los investigadores Teece, Pisano y Shuen (1997), define las capacidades dinámicas como la habilidad que tiene la organización para transformar, las competencias internas y externas, provocando cambios rápidos en el entorno.

Las capacidades dinámicas son adaptables para todo tipo de organización. En el caso particular de las Instituciones de Educación Superior (IES) estas pueden adaptarse y mantenerse en evolución para así afrontar los cambios que producen los nuevos retos generacionales. Los autores Henao et al. (2014) definen las IES conforme al capítulo IV de la Ley 30 de 1992 por el cual se organiza el servicio público de la Educación Superior, expedida por El Congreso de Colombia, aquí se indica que "Son instituciones de educación superior: las instituciones técnicas profesionales, las instituciones universitarias o escuelas tecnológicas y las universidades"; indican que las primeras ofrecen programas de formación en ocupaciones de tipo operativo e instrumental en su respectivo campo de acción; mientras que las instituciones universitarias o escuelas tecnológicas ofrecen programas de formación en ocupaciones, profesiones o disciplinas, y las universidades, además de la formación académica en profesiones o disciplinas, deben acreditar desempeño en investigación científica o tecnológica y la producción, desarrollo y transmisión del conocimiento y de la cultura universal y nacional.

La pertinencia de la actividad investigativa en el interior de las instituciones de educación superior (IES) debe estar enfocada en la medida que estas son aplicadas al mejoramiento de la sociedad (Alvesson y Benner, 2016), es decir utilizar el saber para modificar las actuales condiciones y tratar en la medida de lo posible promover el desarrollo del bienestar social y el desarrollo socio-económico, para ello la IES debería orientar en alguna proporción sus procesos investigativos en los lineamientos o políticas de desarrollo social de orden nacional, regional o local. Con el propósito de identificar las capacidades de aprendizaje que permiten potencializar la investigación en las IES se ha evaluado la interrelación existente entre las variables que las propician, para ello se utiliza un análisis de técnicas estadísticas multivariadas por conglomerados y se corrobora con la técnica de análisis por componentes principales.

El análisis de conglomerados o clúster jerárquico es un método de clasificación de variables. Considera a cada unidad de análisis como un conglomerado, y posteriormente va uniendo los conglomerados de acuerdo con su homogeneidad hasta que queda un conglomerado, entendiendo por grupo homogéneo aquel cuyos miembros difieren significativamente de los de cualquier otro (Sarstedt y Mooi, 2014). El método análisis por componentes principales consiste en la representación de las medidas numéricas de varias variables en un sitio de espacio reducido donde se puedan percibir las relaciones que de otra forma, permanecerían no visibles en dimensiones superiores tiene como objetivo transformar un conjunto de variables originales, en un nuevo conjunto de variables las cuales se caracterizan por estar correlacionadas entre sí (Price et al., 2006).

El aporte de la investigación se evidencia en la determinación de la correlación entre las variables que posibilitan el desarrollo de las capacidades de aprendizaje, lo cual posibilita a las instituciones de educación superior el diseño e implementación de estrategias que conduzcan al mejoramiento de los procesos investigativos

\section{MARCO TEÓRICO}

El origen de las capacidades dinámicas (CD), está basado en la competencia de la innovación de Schumpeter (2017) este afirma que la ventaja competitiva está basada en la reestructuración de los recursos y la adaptación de nuevas capacidades operativas, al respecto Teece (1997) afirma que estas capacidades dinámicas ayudan a dar forma en el diseño del modelo de negocio, pero a su vez este coloca límites en la factibilidad de estrategias particulares, de lo anterior se desprende la posibilidad de adaptación al cambio y a su vez una diferenciación entre los tipos de capacidades dinámicas que se enuncian a continuación en la Tabla 1. 
Tabla 1: Tipos de Capacidades Dinámicas.

\begin{tabular}{|l|l|}
\hline \multicolumn{1}{|c|}{ Capacidad Dinámica } & \multicolumn{1}{c|}{ Descripción } \\
\hline Capacidad dinámica de Absorción & $\begin{array}{l}\text { Se refiere a la posibilidad de asimilar información y } \\
\text { conocimiento externo en función de las posibilidades } \\
\text { presentes Crossan et al. (1999). }\end{array}$ \\
\hline Capacidad dinámica de Innovación & $\begin{array}{l}\text { Está relacionada con la posibilidad de las empresas para } \\
\text { el desarrollo de nuevos productos y la identificación de } \\
\text { nuevos mercados Garzón (2015) }\end{array}$ \\
\hline Capacidad dinámica Adaptación & $\begin{array}{l}\text { Consiste en la posibilidad de realizar ajustes estratégicos } \\
\text { organizacionales para adecuarse a las condiciones } \\
\text { cambiantes del medio (Garzón 2015). }\end{array}$ \\
\hline Capacidad dinámica Aprendizaje & $\begin{array}{l}\text { El desarrollo de esta capacidad se propicia a partir de la } \\
\text { integración de conocimientos individuales y colectivos, } \\
\text { los investigadores Crossan et al. (1999) indican que esto } \\
\text { se genera por la interacción social y la reflexión, interna } \\
\text { y externa de la organización }\end{array}$ \\
\hline
\end{tabular}

\section{Capacidad dinámica de Aprendizaje}

El desarrollo de esta capacidad se propicia a partir de la integración de conocimientos individuales y colectivos. Se favorece al indicar que en el proceso creativo, apropiación, transmisión y uso del conocimiento es posible el crecimiento y fortalecimiento del saber acumulado en medios dinámicos (Garzón, 2015). El autor Vivas, (2013) realiza una recuperación de los trabajos acerca del recurso del conocimiento, indica que es un recurso estratégico para las empresas, esta afirmación es compartida por otros autores como (Grant, 1991) indicando que es un recurso clave para mantener la ventaja competitiva y lograr un desempeño superior. A su vez se comprende esta capacidad como producto de un proceso natural propiciado dentro de las empresas por el aprendizaje colaborativo (Teece et al., 1997; Zollo y Winter, 2002) integrando niveles diversos preexistentes en la organización favoreciendo su desarrollo (Crossan et al., 1999).

El propósito de desarrollar esta capacidad en el interior de la organización es el aprovechamiento de la información proveniente de diversas fuentes por ello es necesario un ambiente proactivo para desarrollar novedosos proyectos, servicios y mejoramiento de procesos, perdurables y adaptables en la organización (Garzón, 2015). Así mismo, los autores Zollo y Winter (2006), resaltan que cuando la organización integra o se adapta rápidamente a los cambios del entorno favorecido por el desarrollo de la capacidad de aprendizaje, esta se convierte realmente en dinámica o adaptable. El desarrollo de las capacidades dinámicas favorece que la institución posea ventajas competitivas puesto que permite gestionar el conocimiento organizativo; el cual puede ser concebido como algo superior a la suma del conocimiento individual de las personas, debido a las sinergias producidas por la interacción entre los individuos y entre éstos y su entorno (Schulz y Jobe 2001).

\section{METODOLOGIA}

En este trabajo, se presenta una metodología para evaluar la correlación existente entre las variables que favorecen el desarrollo de las capacidades de aprendizaje, lo cual posibilita a las instituciones de educación superior potencializar los procesos asociados a la investigación, de conformidad a los fundamentos de investigación propuestos por Michael Crotty (1998), contextualizada en un paradigma positivista, utilizando técnicas cuantitativas de tipo explicativo, con técnicas estadísticas del tipo descriptivas, correlacional e inferencial. Para desarrollar el proceso de evaluación se utiliza el modelo de escala de Likert para un formato de cinco (5) niveles de respuesta como se muestra en la Tabla 2.

Tabla 2: Escala de evaluación de las actividades de validación.

\begin{tabular}{|l|c|}
\hline \multicolumn{1}{|c|}{ Nivel de conformidad } & Valoración \\
\hline Totalmente en desacuerdo & 1 \\
\hline En desacuerdo & 2 \\
\hline Ni de acuerdo ni en desacuerdo & 3 \\
\hline De acuerdo & 4 \\
\hline Totalmente de acuerdo & 5 \\
\hline
\end{tabular}


Inicialmente se toma una muestra de treinta investigadores de diferentes instituciones de educación superior, pertenecientes a grupos de investigación, los cuales evalúan la importancia que poseen para el desarrollo de sus procesos de investigación las variables que se indican a continuación: Trabajo en equipo (TE), Capacitación y formación pos gradual (CP), Políticas institucionales (PI), Alianzas (A), Convenios (C), Redes Académicas (RA) y Vigilancia de Saberes (VS).

A partir de la tabulación de resultados promediados indicados en la Tabla 3 , se utiliza el análisis de conglomerados, el cual es una técnica de análisis multivariado correspondiente a los métodos de clasificación automática o no supervisada, que busca agrupar los elementos de una muestra en grupos homogéneos, teniendo en cuenta la similitud entre ellos. (De la Hoz y Lopez, 2017). Para el análisis se utilizó el software IBM SPSS Statistics 25, este utiliza como criterio de eslabonamiento el método de Ward (Ward, 1963), este es un procedimiento jerárquico en el cual, en cada etapa, se unen los dos clústeres para los cuales se tenga el menor incremento en el valor total de la suma de los cuadrados indica que los conglomerados debían constituirse de tal manera que, al fundirse dos elementos, la pérdida de información resultante de la fusión fuera mínima. Así se cuantifica la cantidad de información como la suma de las distancias al cuadrado de cada elemento respecto al centroide del conglomerado que pertenece, esto es:

$$
\begin{aligned}
& E_{k}=\sum_{i=1}^{n k} \sum_{j=1}^{n}\left(x_{i j}^{k}-m_{j}^{k}\right)^{2} \\
& E=\sum_{k=1}^{h} E_{k}
\end{aligned}
$$

Donde $x_{i j}^{k}$ es j-ésima variable sobre el i-ésimo individuo del k-ésimo clúster, $m_{j}^{k}$ es el valor medio del grupo j, $E_{k}$ la suma de cuadrados de los errores del clúster k, o sea, la distancia euclídea al cuadrado entre cada individuo del clúster $\mathrm{k}$ a su centroide, E es la suma de cuadrados de los errores para todos los clústeres, si se supone que hay h clústeres.

\begin{tabular}{|c|c|c|c|c|c|c|c|}
\hline \multirow[b]{2}{*}{ Requerimientos } & \multicolumn{7}{|c|}{ Variables } \\
\hline & $T E$ & $C P$ & $P I$ & $A$ & $C$ & $R A$ & VS \\
\hline 1. Formulación de proyectos de investigación & 4,52 & 4,93 & 4,97 & 4,83 & 4,83 & 4,43 & 4,43 \\
\hline 2. Vigilancia de los saberes & 3,97 & 3,70 & 2,03 & 3,07 & 1,73 & 3,57 & 5,00 \\
\hline 3. Manejo de metodologías de investigación & 3,23 & 3,90 & 3,00 & 3,07 & 2,23 & 2,67 & 2,97 \\
\hline 4. Relacionamiento & 4,97 & 4,00 & 4,23 & 4,27 & 4,80 & 4,93 & 2,27 \\
\hline 5. Redes académicas & 4,17 & 3,23 & 4,40 & 4,80 & 4,37 & 4,93 & 3,10 \\
\hline $\begin{array}{l}\text { 6. Redacción de artículos científicos, } \\
\text { libros y capítulos de libros }\end{array}$ & 4,20 & 4,73 & 4,00 & 4,03 & 4,10 & 3,90 & 3,87 \\
\hline $\begin{array}{l}\text { 7. Publicación de artículos, } \\
\text { libros y capítulos de libros }\end{array}$ & 4,05 & 4,47 & 4,37 & 4,40 & 4,13 & 3,67 & 3,53 \\
\hline 8. Experiencia de los profesores & 3,12 & 3,50 & 4,33 & 4,63 & 4,17 & 3,93 & 4,03 \\
\hline 9. Capacidad de innovación & 4,05 & 4,93 & 2,90 & 4,07 & 4,13 & 3,70 & 3,87 \\
\hline 10. Desarrollo de patentes & 3,80 & 4,47 & 3,70 & 3,60 & 4,03 & 2,77 & 4,23 \\
\hline 11. Innovaciones en la gestión organizacional & 2,78 & 3,77 & 3,03 & 3,57 & 3,90 & 2,93 & 3,33 \\
\hline $\begin{array}{l}\text { 12. Desarrollo de equipos para generar } \\
\text { soluciones - prototipos }\end{array}$ & 3,88 & 4,10 & 3,23 & 3,20 & 3,93 & 2,87 & 3,80 \\
\hline
\end{tabular}

Tabla 3: Variables de incidencia en la formación de la capacidad de aprendizaje

Posteriormente se utiliza la técnica de análisis de componentes principales donde, el primer factor o componente sería aquel que explica una mayor parte de la varianza total, esto es una indicación de la cantidad de información que lleva la variable; el segundo factor explica la mayor parte de la varianza restante, es decir, de la que no explica el primer factor y así sucesivamente (Urrutia y Lemus, 2010). Para evaluar el grado de asociación entre las variables se utiliza el test KMO (Kaiser, Meyer y Olkin), mide la idoneidad de los datos 
para realizar un análisis factorial comparando los valores de los coeficientes de correlación observados con los coeficientes de correlación parcial (Khadhraoui et al., 2017).

Cuanto más cerca de 1 tenga el valor obtenido del test $\mathrm{KMO}$, implica que la relación entre las variables es alta. Si $\mathrm{KMO} \geq 0.9$, el test es muy bueno; notable para $\mathrm{KMO} \geq 0.8$; mediano para $\mathrm{KMO} \geq 0.7$; bajo para $\mathrm{KMO}$ $\geq 0.6$; y muy bajo para $\mathrm{KMO}<0.5$. La prueba de esfericidad de Bartlett evalúa si las muestras provienen de poblaciones con la misma varianza. El modelo es significativo (aceptamos la hipótesis nula, H0) cuando se puede aplicar el análisis factorial (Arpaci, 2016).

Si Sig. (p-valor) $<0.05$ aceptamos H0 (hipótesis nula) entonces se puede aplicar el análisis factorial.

$\mathrm{Si}$ Sig. (p-valor) $>0.05$ rechazamos $\mathrm{HO}$ entonces no se puede aplicar el análisis factorial.

Este desarrollo permite establecer las variables características de los grupos formados a partir de las capacidades de aprendizaje que potencian la investigación en las IES, e identificar cuáles de estas son las más representativas, con el propósito de adoptar acciones que favorezcan el desarrollo de las mismas.

\section{RESULTADOS Y DISCUSIÓN}

A partir de los resultados obtenidos utilizando la información suministrada por los investigadores, indicada en la Tabla 3, se construye un diagrama radial de influencia entre las variables objeto de estudio Figura 1. Se evidencia que existe un nivel de relación entre las variables y los requerimientos que favorecen el desarrollo de la capacidad de aprendizaje, sin embargo es necesario determinar la articulación entre estas e internamente cuales de ellas presentan mayor interacción.
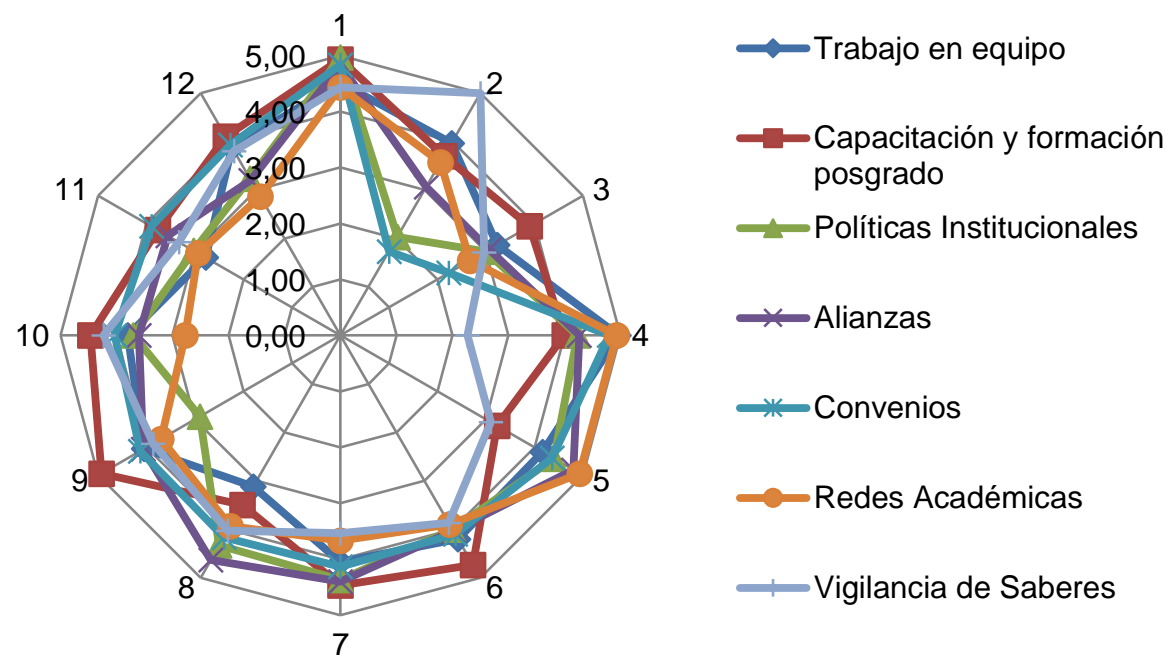

Fig. 1: Incidencia relacional entre requerimientos y variables de investigación

Utilizando el software IBM SPSS Statistics 25, se realiza un análisis de clúster o conglomerados el cual es un método de clasificación de individuos en categorías, a partir de la ecuación (1) y (2) se determina la distancia entre los conglomerados, los resultados se muestran en la Tabla 4.

Tabla 4: Historial de Aglomeración

\begin{tabular}{|c|c|c|c|c|c|c|c|}
\hline Paso & $\begin{array}{r}\text { Número de } \\
\text { conglomerados }\end{array}$ & $\begin{array}{r}\text { Nivel de } \\
\text { semejanza }\end{array}$ & $\begin{array}{l}\text { Nivel de } \\
\text { distancia }\end{array}$ & $\begin{array}{l}\text { Con } \\
\text { inc }\end{array}$ & $\begin{array}{l}\text { ados } \\
\text { dos }\end{array}$ & $\begin{array}{r}\text { Nuevo } \\
\text { conglomerado }\end{array}$ & $\begin{array}{l}\text { Número de obs. En el } \\
\text { conglomerado nuevo }\end{array}$ \\
\hline 1 & 6 & 93,1674 & 0,13665 & 3 & 4 & 3 & 2 \\
\hline 2 & 5 & 88,4595 & 0,23081 & 3 & 5 & 3 & 3 \\
\hline 3 & 4 & 84,1765 & 0,31647 & 1 & 6 & 1 & 2 \\
\hline 4 & 3 & 69,4184 & 0,61163 & 1 & 3 & 1 & 5 \\
\hline 5 & 2 & 63,3839 & 0,73232 & 2 & 7 & 2 & 2 \\
\hline 6 & 1 & 34,7079 & 1,30584 & 1 & 2 & 1 & 7 \\
\hline
\end{tabular}

Se observa que PI (3) y A (4) son las primeras comunidades que se unen en el Historial de Aglomeración, con un nivel de distancia de 0,13665 seguido por $\mathrm{PI}$ (3) y C (5) con un nivel de distancia de 0,23081 , las cuales 
presentan la mayor relación entre variables por ende la menor distancia, de manera similar TE (1) y CP (2) son las variables que presentan la menor relación, por consiguiente una distancia mayor equivalente a 1,30584. Para representar el nivel de semejanza o proximidad que existe entre las variables se utiliza un dendrograma el cual se muestra en la Figura 2, aquellas variables que se encuentren más cercanas se asocian en el mismo conglomerado y las que menor relación posean entre sí, formaran parte de otros conglomerados o clúster, se utilizó el método de Ward - intervalo de distancia Euclídea cuadrado.

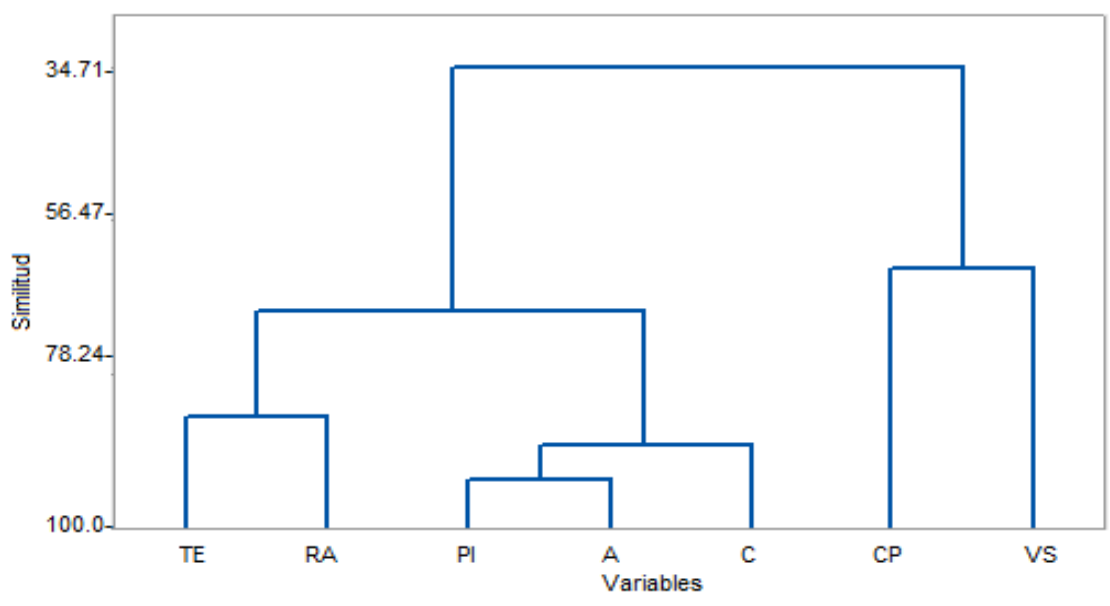

Fig. 2: Dendrograma: Enlace de Ward; Distancia Euclídea cuadrado

Se observa que se forman dos grandes conglomerados, el primero conformado por las variables TE-RA, y PIA-C, y el segundo conformado por CP-VS. Los objetos similares se conectan mediante enlaces cuya posición en el diagrama está determinada por el nivel de similitud/disimilitud entre los objetos. Con el propósito de corroborar el resultado obtenido se realiza una comparación utilizando la técnica de análisis por componentes principales, el cual es un método de reducción de datos, que minimiza la dimensión del número de variables consideradas. En la Tabla 5, se obtiene la varianza total explicada, observándose que para los dos primeros componentes es del orden de $71.85 \%$, lo cual es un indicativo de un buen nivel de relacionamiento entre las dos variables del conglomerado.

Tabla 5: Varianza total explicada

\begin{tabular}{|r|r|r|r|r|r|r|r|r|r|}
\hline \multirow{2}{*}{ Componente } & \multicolumn{3}{|c|}{ Autovalores iniciales } & \multicolumn{4}{|c|}{$\begin{array}{c}\text { Sumas de cargas al cuadrado } \\
\text { de la extracción }\end{array}$} & \multicolumn{3}{|c|}{$\begin{array}{c}\text { Sumas de cargas al cuadrado de } \\
\text { la rotación }\end{array}$} \\
\cline { 2 - 11 } & Total & $\begin{array}{c}\% \text { de } \\
\text { varianza }\end{array}$ & $\begin{array}{c}\% \\
\text { acumulado }\end{array}$ & Total & $\begin{array}{c}\% \text { de } \\
\text { varianza }\end{array}$ & $\begin{array}{c}\% \\
\text { acumulado }\end{array}$ & Total & $\begin{array}{c}\% \text { de } \\
\text { varianza }\end{array}$ & $\begin{array}{c}\% \\
\text { acumulado }\end{array}$ \\
\hline 1 & 3,656 & 52,232 & 52,232 & 3,656 & 52,232 & 52,232 & 3,656 & 52,227 & 52,227 \\
\hline 2 & 1,373 & 19,621 & 71,853 & 1,373 & 19,621 & 71,853 & 1,374 & 19,626 & 71,853 \\
\hline 3 & 0,840 & 12,006 & 83,859 & & & & & & \\
\hline 4 & 0,775 & 11,072 & 94,931 & & & & & & \\
\hline 5 & 0,178 & 2,544 & 97,475 & & & & & & \\
\hline 6 & 0,160 & 2,283 & 99,758 & & & & & & \\
\hline 7 & 0,017 & 0,242 & 100,000 & & & & & & \\
\hline
\end{tabular}

La idoneidad de los datos obtenidos es verificada utilizando la prueba de Kaiser-Meyer-Olkin (KMO), en la Tabla 6, se observan los resultados alcanzados.

Tabla 6: Prueba de KMO y Bartlett

\begin{tabular}{|l|l|r|}
\hline \multicolumn{2}{|l|}{ Medida Kaiser-Meyer-Olkin de adecuación de muestreo } & 0,445 \\
\hline \multirow{2}{*}{$\begin{array}{l}\text { Prueba de esfericidad de } \\
\text { Bartlett }\end{array}$} & Aprox. Chi-cuadrado & 50.552 \\
\cline { 2 - 3 } & $\mathrm{gl}$ & 21 \\
\cline { 2 - 3 } & Sig. & 0,000 \\
\hline
\end{tabular}


De los datos obtenidos se infiere que el análisis de componentes principales es factible debido a que el valor p-valor (Sig.) $<0,05$, sin embargo la correlación entre todas las variables es baja (el valor obtenido por el test de $\mathrm{KMO}$ es 0,531 ). Esto es un indicativo que existe relaciones parciales entre las variables pero no en la totalidad de ellas. En la Tabla 7, se muestra la matriz de componente rotado, en la que se utiliza el método de rotación Varimax con normalización Kaiser. Se observa que existe una alta correlación lineal entre A-PI$\mathrm{C}$, y una menor relación de estos con RA-TE, adicionalmente una media correlación lineal entre CP-VS. Conforme a esto se entiende que la agrupación de las variables se da en dos componentes:

a) Componente 1: la asociación de las variables Alianza con un $91,3 \%$, Convenios con un $86,2 \%$ y Redes académicas con un $84,0 \%$ indica que existe una alta relación entre el sector externo y la institución, además la institución favorece este relacionamiento por medio de las políticas institucionales con un $88.8 \%$, así mismo el Trabajo en Equipo representa un $66.0 \%$ lo cual indica que deben existir mecanismos favorables para el desarrollo de la capacidad de aprendizaje que favorece la interacción interna y externa asociada al desarrollo de la investigación.

b) Componente 2: la asociación entre Capacitación y Estudios Pos graduales $87.9 \%$ y la Vigilancia de Saberes $66.9 \%$ indica que se requieren unas capacidades previas adquiridas y el monitoreo del sector en el campo de interés para favorecer el desarrollo de la capacidad de aprendizaje.

Para indicar la dispersión de estas asociaciones lineales y la relación no lineal entre VS con A-PI-RA, se utiliza la Figura 3 , se aplica la rotación Varimax, la cual consigue que cada componente rotado presente correlaciones entre las variables.

Tabla 7: Matriz de componente rotado

\begin{tabular}{|c|c|c|}
\hline & \multicolumn{2}{|c|}{ Componente } \\
\hline & 1 & 2 \\
\hline $\mathrm{A}$ & 0,913 & $-0,065$ \\
\hline $\mathrm{PI}$ & 0,888 & $-0,048$ \\
\hline $\mathrm{C}$ & 0,862 & 0,053 \\
\hline $\mathrm{RA}$ & 0,840 & $-0,177$ \\
\hline TE & 0,660 & 0,337 \\
\hline CP & 0,222 & 0,879 \\
\hline VS & $-0,316$ & 0,669 \\
\hline
\end{tabular}

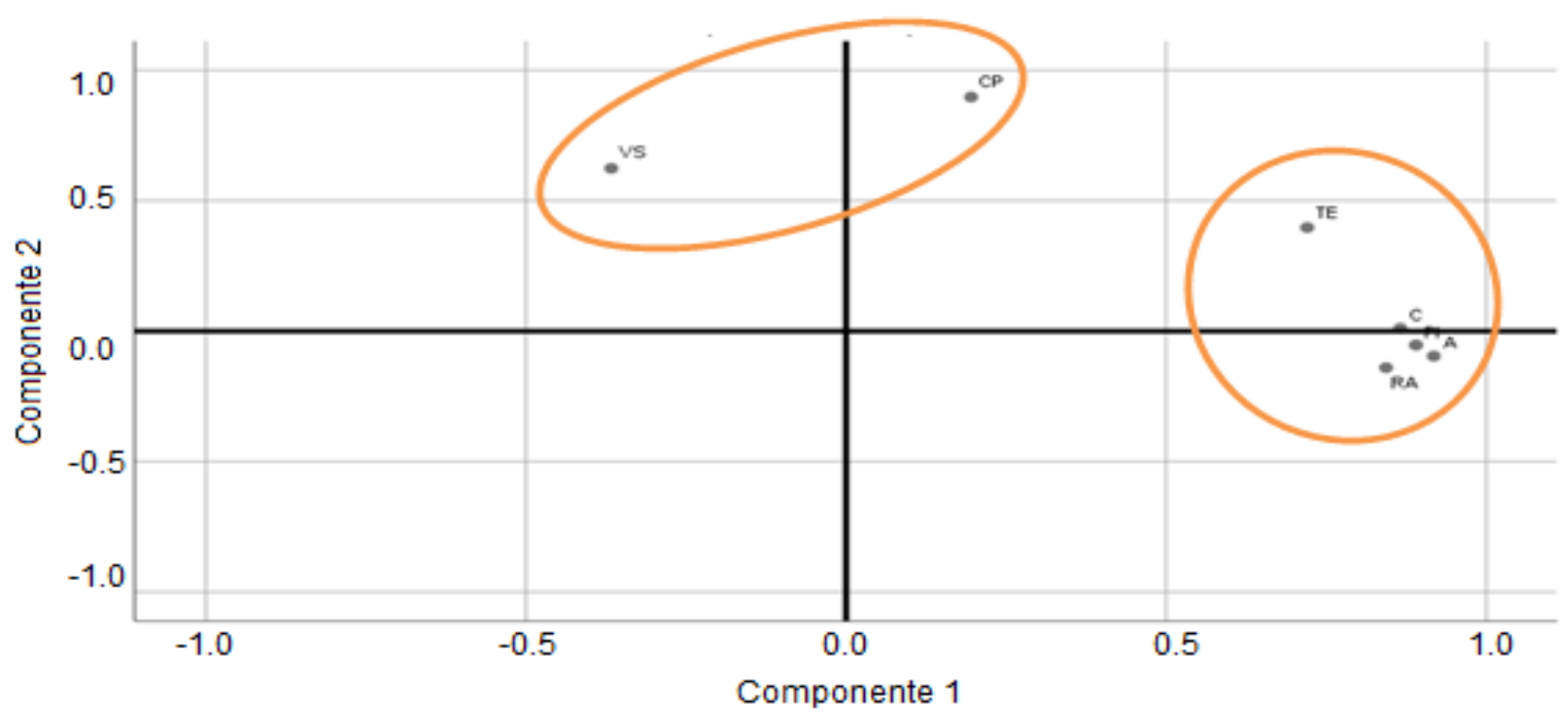

Fig. 3: Componentes en espacio rotado 


\section{CONCLUSIONES}

Con base en los resultados obtenidos y su discusión, es posible obtener las siguientes conclusiones:

1. Se logró determinar la relación existente entre los requerimientos necesarios para potenciar la capacidad de aprendizaje que fomenta la investigación en las IES y las variables propuestas, 2 . Se utiliza una técnica de análisis multivariado para la identificación de las variables que presentan mayor nivel de relacionamiento en función del desarrollo de la capacidad de aprendizaje, 3. La técnica de análisis multivariado por conglomerados o clúster demostró ser efectiva para la identificación de las variables relacionadas, a su vez los resultados obtenidos fueron corroborados con la técnica de análisis de componentes principales

\section{AGRADECIMIENTOS}

Los autores expresan su agradezco a la Dirección de Investigaciones de la Universidad Cooperativa de Colombia Sede Ibagué - Espinal y a la Dirección de Investigaciones de la Universidad de lbagué, por el apoyo suministrado para el desarrollo de la presente investigación.

\section{REFERENCIAS}

Alvesson, M. y M. Benner, Higher Education in the knowledge society: Miracle or Mirage? Multi-level governance in universities, Springer, Cham, 75-91 (2016)

Arpaci, I., Understanding and Predicting Students Intention to Use Mobile Cloud Storage Services, Computers in Human Behavior, 58, 150-157 (2016)

Crossan, M.M., H.W. Lane y R.E. White, An Organizational Learning Framework: From Intuition to Institution, Academy of Management Review, 24(3), 522-537(1999)

Crotty, M., The Foundations of Social Research: Meaning and Perspective, Research Process, Sage Publications London (1998)

De La Hoz, E. y L. López Polo, Aplicación de Técnicas de Análisis de Conglomerados y Redes Neuronales Artificiales en la Evaluación del Potencial Exportador de una Empresa, Información Tecnológica, 28(4), 67-74 (2017)

Garzón Castrillón, M.A., Modelo de Capacidades Dinámicas, Dimensión Empresarial, 13(1), 111-131 (2015)

Grant, R. M., The Resource-Based Theory of Competitive Advantage: Implications for Strategy Formulation, California Management Review, 33(3), 114-135 (1991)

Helfat, C. y M. Peteraf, Managerial Cognitive Capabilities and the Microfoundations of Dynamic Capabilities, Strategic Management Journal, 36(6), 831-850 (2015)

Henao, E.A., M. López y R. Garcés, Medición de Capacidades en Investigación e Innovación en Instituciones de Educación Superior: Una Mirada Desde el Enfoque de las Capacidades Dinámicas, Entramado, 10(1), (2014)

Khadhraoui, M., M. Plaisentm, P. Bernard y L. Lakhal, The Impact of Marketing Skills and Negotiation Skills of Universities Technology Transfer Office on Technology Transfer Success, Journal of Marketing and Management, 8(2), 38-46 (2017)

Price, A.L., N.J. Patterson y otros cuatro autores, Principal Components Analysis Corrects for Stratification in GenomeWide Association Studies, Nature Genetics, 38(8), 904, (2006)

Sarstedt, M. y E. Mooi, Cluster Analysis. In a Concise Guide to Market Research, Springer, Berlin, Heidelberg, 273-324 (2014)

Schulz, M. y L.A. Jobe, Codification and Tacitness as Knowledge Management Strategies: An Empirical Exploration, The Journal of High Technology Management Research, 12(1), 139-165 (2001)

Schumpeter, J.A., Theory of Economic Development, Routledge, New York (2017)

Teece, D., G. Pisano y A. Shuen, Dynamic Capabilities and Strategic Management, Strategic Management Journal, 18(7), 509-533 (1997)

Urrutia, J. A. y R.P. Lemus, Componentes Principales en la Determinación de Estaciones con Patrones Homogéneos de Temperatura en el Chocó, Scientia et Technica, 2(45), 257-262 (2010)

Vivas López, S., Implicaciones de las Capacidades Dinámicas para la Competitividad y la Innovación en el Siglo XXI, Cuadernos de Administración, 26(47), 119-139 (2013)

Ward, J.H., Hierarchical Grouping to Optimize an Objective Function, Journal of the American Statistical Association, 58, 236-244 (1963)

Zollo, M. y S.G. Winter, Deliberate Learning and the Evolution of Dynamic Capabilities, Organization Science, 13(3), 339$351(2002)$ 